\title{
Análise das propriedades phase-lag e erro de amplificação do método RK-Butcher
}

\author{
Heloisa H. M. Silva \\ Depto de Matemática Aplicada, IBILCE, UNESP, \\ 15054-000, São José do Rio Preto, SP \\ E-mail: hsilva@ibilce.unesp.br
}

Resumo: Consideramos, neste trabalho, as propriedades phase-lag e erro de amplificação de um método embutido de Runge-Kutta, denominado algoritmo RK-Butcher [2], para problemas de valor inicial que apresentam soluções oscilatórias e verificamos sua eficiência através de problemas testes. As propriedades de estabilidade deste método para problemas de valor inicial de primeira ordem foram analisadas em [5].

Palavras-chave: Equações diferencias ordinárias, métodos de Runge-Kutta, problemas oscilatórios.

\section{Introdução}

Neste trabalho estamos interessados na resolução numérica de problemas de valor inicial de primeira ordem da forma

$$
y^{\prime}=f(t, y), \quad y\left(t_{0}\right)=\eta,
$$

que possuem soluções oscilatórias. Para estes tipos de problemas é essencial considerarmos as propriedades phase-lag (ou dispersão) e amplificação (ou dissipação). Estas propriedades são, na verdade, dois tipos de erros de truncamento. O primeiro é o ângulo entre a solução verdadeira e a solução aproximada enquanto o segundo é a distância de uma solução cíclica padrão. Aplicações dos métodos de Runge-Kutta (RK) para solução de problemas do tipo (1) foram primeiramente considerados por Brusa e Nigro[1] que introduziram a propriedade phaselag como uma ferramenta para analisar o comportamento dos métodos para problemas com soluções oscilatórias. Desde então, vários trabalhos voltados para análise e desenvolvimento de métodos do tipo explícito e implícito para equações diferencias ordinárias de primeira e segunda ordem, foram publicados, entre os quais citamos Van der Houwen e Sommeijer [9], [10], Calvo e outros [3], Chawla e Al-Zanaid [4], Senu e outros [6].

\section{Erros de Dispersão e Amplificação}

Para examinar as propriedades de estabilidade de métodos numéricos para a resolução de problemas oscilatórios do tipo (1), a equação teste relevante é dada por

$$
y^{\prime}=i \omega y, \omega \in \Re, i=\sqrt{-1},
$$

cuja solução exata é $y(t)=\exp (i \omega t)$.

Um método geral de Runge-Kutta explícito, com $s$ estágios para resolução de problemas de valor inicial de primeira ordem dados pela equação (1) é definido por

$$
y_{n+1}=y_{n}+\sum_{i=1}^{s} b_{i} K_{i},
$$




$$
K_{i}=f\left(x_{n}+c_{i} h, y_{n}+h \sum_{j=1}^{s} a_{i j} K_{j}\right),
$$

$\operatorname{com} c_{i}=\sum_{j=1}^{s} a_{i j}, i=1,2, \ldots, s, c$ e $b$ vetores $s$-dimensionais e $A=\left(a_{i j}\right)$ uma matriz $s \times s$. Usando a notação matricial de Butcher o método é escrito como

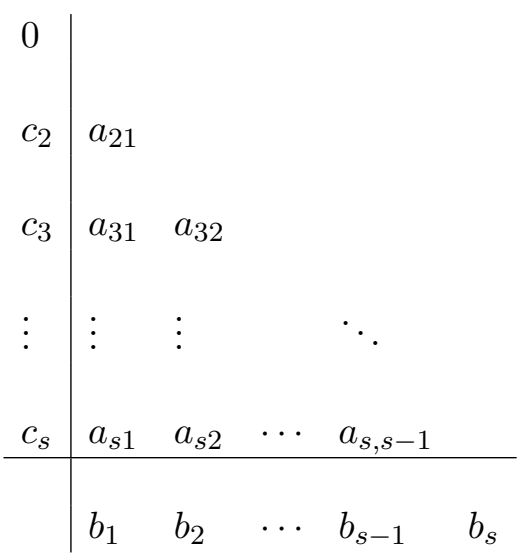

Assim, aplicando o método de Runge-Kutta ao problema teste (2) obtém-se a solução numérica

$$
y_{n}=a_{s}^{n} y_{0}, a_{s}=A_{s}\left(H^{2}\right)+i H B_{s}\left(H^{2}\right),
$$

onde $h=\omega H, A_{s}$ e $B_{s}$ são polinômios em $H^{2}$ definidos pelos parâmetros $c_{i}, a_{i, j}$ e $b_{i}, i=$ $1, \ldots, s, j=1, \ldots, i-1$. O fator de amplificação é $a_{s}=a_{s}(H)$, e $y_{n}$ denota a aproximação para $y\left(t_{n}\right)$.

Através de uma comparação da solução numérica (5) com a solução de (2), dada por $y\left(t_{n}\right)=y_{0} \exp (i n H)$, Sideridis e Simos [7] definem os conceitos e resultados apresentados a seguir.

Definição 1 Denomina-se, respectivamente, erro de dispersão (ou phase-lag) e erro de amplificação do método de Runge-Kutta (4) às quantidades:

$$
p l(H)=H-\arg \left[a_{s}(H)\right], \quad a(H)=1-\left|a_{s}(H)\right|
$$

Definição 2 Se $p l(H)=O\left(H^{r+1}\right)$ e $a(H)=O\left(H^{s+1}\right)$, então diz-se que o método de RungeKutta (4) tem, respectivamente, ordem phase-lag $r$ e ordem dissipativa $q$.

Teorema 1 Se o método de Runge-Kutta dado por (4), (5) possui ordem phase-lag r então:

$$
\tan H-\frac{H B_{s}\left(H^{2}\right)}{A_{s}\left(H^{2}\right)}=c H^{r+1}+O\left(H^{r+3}\right)
$$

onde c é a constante phase-lag do método.

Como consequência desse resultado temos que se o método de Runge-Kutta tem ordem phase-lag $r$, então podemos reescrever $p l(H)$ como

$$
p l(H)=\tan (H)-\frac{H B_{s}\left(H^{2}\right)}{A_{s}\left(H^{2}\right)} .
$$




\section{O método RK-Butcher}

O método RK-Butcher é um método embutido de Runge-Kutta, isto é, um par de fórmulas de Runge-Kutta, com 6 estágios, que aplicado ao problema (1) produz duas aproximações $y_{n+1}$ e $y_{n+1}^{*}$ para $y\left(t_{n+1}\right)\left(t_{n+1}=t_{n}+h\right)$, de ordens algébricas $p=5$ e $p^{*}=4$, respectivamente, onde

$$
\begin{aligned}
y_{n+1}= & y_{n}+\frac{1}{90}\left(7 K_{1}+32 K_{3}+12 K_{4}\right. \\
& \left.+32 K_{5}+7 K_{6}\right), \\
y_{n+1}^{*}= & y_{n}+\frac{1}{6}\left(K_{1}+4 K_{4}+K_{6}\right), \\
K_{1}= & h f\left(x_{n}, y_{n}\right), \\
K_{2}= & h f\left(x_{n}+\frac{h}{4}, y_{n}+\frac{K_{1}}{4}\right) \\
K_{3}= & h f\left(x_{n}+\frac{h}{4}, y_{n}+\frac{K_{1}}{8}+\frac{K_{2}}{8}\right) \\
K_{4}= & h f\left(x_{n}+\frac{h}{2}, y_{n}-\frac{K_{2}}{2}+K_{3}\right) \\
K_{5}= & h f\left(x_{n}+\frac{3 h}{4}, y_{n}+\frac{3 K_{1}}{16}+\frac{9 K_{4}}{16}\right) \\
K_{6}= & h f\left(x_{n}+h, y_{n}-\frac{3 K_{1}}{7}+\frac{2 K_{2}}{7}+\frac{12 K_{3}}{7}\right. \\
& \left.-\frac{12 K_{4}}{7}+\frac{8 K_{5}}{7}\right) .
\end{aligned}
$$

Usando a notação matricial de Butcher o método (8) é escrito na forma

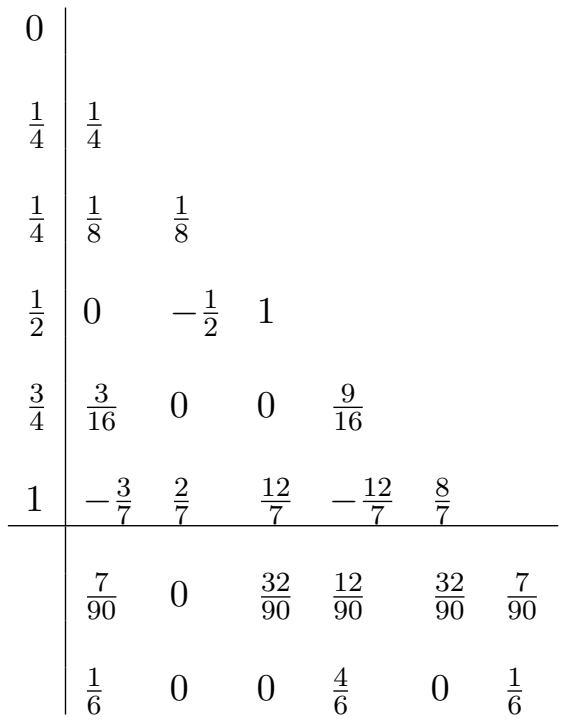

Aplicando o método RK-Butcher (9), (10) à equação teste (2) nós temos (5) com

$$
a_{6}^{*}=A_{6}^{*}\left(H^{2}\right)+i H B_{6}^{*}\left(H^{2}\right),
$$

onde

$$
\begin{gathered}
A_{6}^{*}\left(H^{2}\right)=1-\frac{1}{2} H^{2}+\frac{1}{21} H^{4}-\frac{3}{896} H^{6}, \\
B_{6}^{*}\left(H^{2}\right)=1-\frac{1}{6} H^{2}+\frac{1}{224} H^{4} .
\end{gathered}
$$

Agora, aplicando (11) em (7), obtemos:

$$
\begin{aligned}
p l^{*}(H) & =\tan (H)-H \frac{B_{6}^{*}\left(H^{2}\right)}{A_{6}^{*}\left(H^{2}\right)} \\
& =\frac{11}{1120} H^{5}+O\left(H^{7}\right) . \\
010062-3 &
\end{aligned}
$$


De (11) e da Definição 1, encontramos:

$$
a^{*}(H)=-\frac{1}{168} H^{4}+O\left(H^{6}\right) .
$$

Da Definição 2 e dos resultados (12) e (13), concluímos que o método RK-Butcher de ordem 4 tem ordem phase-lag 4 e ordem dissipativa 3.

Usando o mesmo raciocínio, analisamos, em seguida, as propriedades do método RK-Butcher de ordem algébrica 5 dado por (8) e (10). Aplicando este método à equação teste (1) obtemos

$$
a_{6}=A_{6}\left(H^{2}\right)+i H B_{6}\left(H^{2}\right),
$$

onde

$$
\begin{gathered}
A_{6}\left(H^{2}\right)=1-\frac{1}{2} H^{2}+\frac{1}{24} H^{4}-\frac{1}{640} H^{6}, \\
B_{6}\left(H^{2}\right)=1-\frac{1}{6} H^{2}+\frac{1}{120} H^{4} .
\end{gathered}
$$

e phase-lag igual a

$$
\begin{aligned}
p l(H) & =\tan (H)-H \frac{B_{6}\left(H^{2}\right)}{A_{6}\left(H^{2}\right)} \\
& =-\frac{1}{2688} H^{7}+O\left(H^{9}\right) .
\end{aligned}
$$

Logo, temos que

$$
a(H)=-\frac{1}{5760} H^{6}+O\left(H^{8}\right)
$$

Assim, o método de ordem 5 tem ordem phase-lag 6 e ordem dissipativa 5.

\section{Resultados Numéricos}

Apresentamos, nesta seção, os resultados numéricos obtidos pelo algoritmo RK-Butcher em um problema modelo dado pela equação

$$
y^{\prime}=\left[\begin{array}{rr}
0 & \phi \\
-\phi & 0
\end{array}\right] y
$$

com condição inicial $y(0)=\left(\begin{array}{ll}1 & 0\end{array}\right)^{T}$. A solução exata é dada por

$$
y=\left[\begin{array}{r}
\cos (\phi x) \\
-\operatorname{sen}(\phi x)
\end{array}\right] .
$$

Na Tabela 1 apresentamos os erros absolutos máximos, no intervalo $[0,10]$, para $\phi=5$ e usando tamanho do passo $h=1 / 8$ e $h=1 / 16$, onde $y_{1}(x)$ e $y_{2}(x)$ representam as componentes da solução $y(x)$ e $y_{1, j}, y_{2, j}$ as correspondentes soluções numéricas. Observamos neste problema modelo que o método apresenta resultados satisfatórios e mostra-se adequado para problemas com soluções oscilatórias. Pretendemos aplicar o método em outros tipos de problemas oscilatórios e também fazer sua implementação usando passo variável aproveitando sua estrutura de um método embutido. 


\begin{tabular}{ccc}
$h$ & $\max \left|y_{1}\left(x_{j}\right)-y_{1, j}\right|$ & $\max \left|y_{2}\left(x_{j}\right)-y_{2, j}\right|$ \\
\hline $1 / 8$ & $9.90129 \times 10^{-4}$ & $1.04902 \times 10^{-3}$ \\
$1 / 16$ & $2.65702 \times 10^{-5}$ & $2.74461 \times 10^{-5}$
\end{tabular}

Tabela 1: erro absoluto máximo no intervalo $[0,10]$ para $\mathrm{h}=1 / 8$ e $\mathrm{h}=1 / 16$

\section{Referências}

[1] L. Brusa and L. Nigro, A one step method for direct integration of structural dynamic equations, Internat. J. Numer. Methods Eng., 15 (1980) 685-699.

[2] M. Bader, A new technique for the early detection of stiffness in coupled differential equations and application to standard Runge-Kutta algorithms, Theoretical Chemistry Accounts, 99 (1998) 215-219.

[3] M. Calvo, J.M. Franco, J.I. Montijano, L. Randéz, Explicit Runge-Kutta methods for initial value problems with oscillating solutions, J. Comput. Appl. Math., 76 (1996) 195-212.

[4] M.M. Chawla, M.A. Al-Zanaid, Non-dissipative extended one-step methods for oscillatory problems, Int. J. Comput. Math., 69 (1998) 85-100.

[5] K. Murugesan, S. Sekar, V. Murugesh and D.J. Evans, Numerical strategies for the system of first-order IVPs using the RK-Butcher algorithm, Internat. J. Comput. Math., 82 (2005) 1379-1387.

[6] N. Senu, M. Suleiman, F. Ismail, and M. Othman, A Singly Diagonally Implicit RungeKutta-Nyström Method for Solving Oscillatory Problems, IAENG International Journal of Applied Mathematics, 41 (2011) 155-161.

[7] A.B. Sideridis and T.E. Simos, A low-order embedded Runge-Kutta method for periodic initial-value problems, J. Comput. Appl. Math., 44 (1992) 235-244.

[8] T.E. Simos, A two-step method with phase-lag of order infinity for the numerical integration of second order periodic initial-value problems, Internat. J. Comput. Math., 39 (1991) 135140 .

[9] P.J. Van der Houwen and B.P. Sommeijer, Explicit Runge-Kutta (Nystrom) methods with reduced phase errors for computing oscillating solutions, SlAM J. Numer. Anal., 24 (1987) 595-617.

[10] P.J. Van der Houwen and B.P. Sommeijer, Phase-lag analysis of implicit Runge-Kutta methods, SlAM J. Numer. Anal., 26 (1989) 214-229. 